\title{
Acetylation of Lysine 243 Inhibits the oriC Binding Ability of DnaA in Escherichia coli
}

\author{
Shuxian Li', Qiufen Zhang ${ }^{1}$, Zhihong $X u^{1}$ and Yu-Feng Yao ${ }^{1,2 *}$ \\ 1 Laboratory of Bacterial Pathogenesis, Institutes of Medical Sciences, Shanghai Jiao Tong University School of Medicine, \\ Shanghai, China, ${ }^{2}$ Department of Laboratory Medicine, Shanghai East Hospital, Tongji University School of Medicine, \\ Shanghai, China
}

OPEN ACCESS

Edited by:

Xihui Shen,

Northwest A\&F University, China

Reviewed by:

Wenxing Liang,

Qingdao Agricultural University, China Alan J. Wolfe,

Loyola University Chicago, USA

${ }^{*}$ Correspondence: Yu-Feng Yao yfyao@sjtu.edu.cn

Specialty section:

This article was submitted to Infectious Diseases,

a section of the journal

Frontiers in Microbiology

Received: 28 February 2017 Accepted: 05 April 2017

Published: 20 April 2017

Citation:

Li S, Zhang Q, Xu Z and Yao Y-F (2017) Acetylation of Lysine 243 Inhibits the oriC Binding Ability of DnaA in Escherichia coli.

Front. Microbiol. 8:699. doi: 10.3389/fmicb.2017.00699
DNA replication initiation is a central event in the cell cycle, and it is strictly controlled by multiple regulatory mechanisms. Our previous work showed that acetylation of residue lysine (K) 178 prevents DnaA from binding to ATP, which leads to the inhibition of DNA replication initiation. Here, we show that another residue, K243, is critical for DnaA full activity in vivo. K243 can be acetylated, and its acetylation level varies with cell growth. A homogeneous, recombinant DnaA that contains $\mathrm{N}^{\varepsilon}$-acetyllysine at K243 (K243Ac) retained its ATP/ADP binding ability, but showed decreased binding activity to the oriC region. A DNase I footprinting assay showed that DnaA K243Ac failed to recognize DnaA boxes I3, C1, and C3, and, thus, it formed an incomplete initiation complex with oriC. Finally, we found that acetyl phosphate and the deacetylase CobB can regulate the acetylation level of K243 in vivo. These findings suggest that DnaA K243 acetylation disturbs its binding to low-affinity DnaA boxes, and they provide new insights into the regulatory mechanisms of DNA replication initiation.

Keywords: Iysine acetylation, replication initiation, initiator DnaA, acetyl-phosphate, Escherichia coli

\section{INTRODUCTION}

Chromosome replication initiation is a central event in the bacterial cell cycle, and the initiator DnaA plays essential roles in this process (Kornberg and Baker, 1992; Messer, 2002; Mott and Berger, 2007). In Escherichia coli, DnaA forms complex with ATP/ADP, and it binds to the replication origin (oriC). This interaction leads to a local unwinding in an adjacent AT-rich region, which is assisted by the DNA-structuring proteins $\mathrm{HU}$ or integration host factor (IHF) (Hwang and Kornberg, 1992). The unwound region provides an entry site for the DnaB-DnaC helicase complex in a manner mediated by DnaC and an oriC-bound DnaA interaction. Then, DnaB migrates along the DNA to expand the region of single-stranded DNA (Kornberg and Baker, 1992), which leads to the assembly of replication machineries, including the DnaG primase and the DNA polymerase III holoenzyme (O'Donnell, 2006).

Binding of DnaA to the replication origin is the first step of nascent DNA synthesis in all systems (Baker and Bell, 1998). oriC of E. coli contains, within $260 \mathrm{bp}$, five copies of 9-mer DnaA binding sites (R boxes, R1-R5) with the consensus sequence 5'-TT(A/T)TNCACA-3' (Schaper and Messer, 1995). Although differing slightly in their nucleotide sequences, these DnaA boxes have different binding affinities for DnaA (Sekimizu et al., 1987; Schaper and Messer, 1995). Speck and Messer (2001) found that ATP-DnaA but not ADP-DnaA adopts a new binding specificity for the consensus sequence $5^{\prime}-\mathrm{AG}(\mathrm{A})(\mathrm{T})(\mathrm{C})(\mathrm{T})-3^{\prime}$. These 6-mer ATP-DnaA boxes located in 
the AT-rich DNA-unwinding element (DUE) are bound cooperatively by ATP-DnaA, which promotes unwinding of the DUE region (Speck and Messer, 2001). ATP-DnaA also binds to another class of sequence flanking the center of oriC, termed I sites, which have the consensus sequence $5^{\prime}-(\mathrm{A} / \mathrm{T})$ $\mathrm{G}(\mathrm{G} / \mathrm{C})(\mathrm{A} / \mathrm{T}) \mathrm{N}(\mathrm{G} / \mathrm{C}) \mathrm{G}(\mathrm{A} / \mathrm{T})(\mathrm{A} / \mathrm{T})(\mathrm{T} / \mathrm{C}) \mathrm{A}-3^{\prime}$ (Grimwade et al., 2000; McGarry et al., 2004). Subtly different from the R box, DnaA interactions with these sites are enhanced in the presence of IHF. Although with low affinity for ATP-DnaA binding, I sites are required for DNA strand opening (Grimwade et al., 2000; McGarry et al., 2004). Furthermore, Kawakami et al. (2005) found $\tau$ sites ( $\tau 1$ and $\tau 2$ ) sharing sequence homology with the I2 and I3 sites, while Rozgaja et al. (2011) reported C sites (C1, C2, and C3). All the low-affinity sites together provide nucleotide-based instructions for the ordered assembly of DnaA oligomers (Rozgaja et al., 2011). DnaA interaction with oriC is cooperative, and it occurs in a specific order, with high-affinity sites (R4, R1, and R2) filled throughout most of the cell cycle, while low-affinity sites are only filled immediately before the onset of DNA synthesis (Ryan et al., 2004). DnaA binding to low-affinity sites is more important in triggering initiation, but the key DnaA residues that are responsible for recognizing low-affinity sites are less understood.

As the genetic information carrier, the replication of DNA must be limited to once per cell cycle, and it must occur at the correct time. Multiple mechanisms regulate this process at the initiation stage (Mott and Berger, 2007; Katayama et al., 2010). Our previous work showed that acetylation of DnaA at the conserved lysine $(\mathrm{K})$ residue 178 in the Walker A motif inhibited its ATP/ADP binding ability and its subsequent oriC binding activity (Zhang et al., 2016). In this study, we found that acetylation of $\mathrm{K} 243$ inhibits the oriC binding activity of DnaA, but it does not affect the ATP/ADP binding affinity of DnaA or the ability of DnaA to bind the $d_{n a A}$ promoter region and the DnaA-reactivating sequence 1 (DARS1). Our data suggest that acetylation negatively regulates DNA replication initiation by disturbing the binding of DnaA to low-affinity boxes, which increases our understanding of this precise replication initiation process.

\section{MATERIALS AND METHODS}

\section{Bacterial Strains, Plasmids, Primers, and Media}

All the bacterial strains, plasmids, and primers used in this study are listed in Supplementary Tables S1, S2.

\section{Protein Purification Procedure}

The site-specific acetylated DnaA (K243Ac) was purified as previously described (Ren et al., 2016; Zhang et al., 2016) with some modifications. $1 \mathrm{~L}$ culture of E. coli strain BL21 carrying plasmid pAcKRS-3 and pCDF-PylT- $d$ naA (K243TAG) was grown at $37^{\circ} \mathrm{C}$ in $\mathrm{LB}$ medium supplemented with streptomycin $(50 \mu \mathrm{g} / \mathrm{mL})$ and kanamycin $(50 \mu \mathrm{g} / \mathrm{mL})$. At an $\mathrm{OD}_{600}=1.6-1.8$, $1 \mathrm{~L} \mathrm{LB}$ with $20 \mathrm{mM}$ acetyl-lysine (AcK) was added. DnaA K243Ac was induced overnight by adding IPTG at a final concentration of $1 \mathrm{mM}$.

The harvested cells were resuspended in cold buffer A $(50 \mathrm{mM}$ Tris- $\mathrm{HCl}, \mathrm{pH}$ 7.5, $500 \mathrm{mM} \mathrm{NaCl}, 20 \mathrm{mM}$ imidazole, $10 \%$ glycerol), cell suspension was lysed by pressure cell disrupters. The lysate was centrifuged to collect the supernatant, ammonium sulfate was added slowly at a final concentration of $0.22 \mathrm{~g} / \mathrm{ml}$, and the resulting precipitate was collected by centrifugation, dissolved with buffer A. After dialyzed overnight against the same buffer, the solution was loaded to a $1 \mathrm{ml} \mathrm{Ni-NTA} \mathrm{column} \mathrm{(GE}$ Healthcare) pre-washed with buffer A. After washing with buffer $\mathrm{A}$, the protein was eluted with buffer B $(50 \mathrm{mM}$ Tris- $\mathrm{HCl}, \mathrm{pH}$ 7.5, $500 \mathrm{mM} \mathrm{NaCl}, 500 \mathrm{mM}$ imidazole, $10 \%$ glycerol), fractions containing DnaA K243Ac were desalted using a $5 \mathrm{ml}$ HiTrap desalting column (GE Healthcare) pre-washed with buffer D (50 mM HEPES-KOH, pH7.5, 0.1 mM EDTA, 20\% sucrose, $2 \mathrm{mM}$ DTT, $0.2 \mathrm{M}$ ammonium sulfate, $10 \mathrm{mM}$ magnesium). The wild-type DnaA was purified in the same way, protein at purity $>90 \%$ (Supplementary Figure S1) was kept frozen at $-80^{\circ} \mathrm{C}$.

The purification of YfiQ, CobB and native DnaA proteins were the same as previously described (Zhang et al., 2016).

\section{Plasmid Complementation Assay}

This assay was performed mainly as described (Kawakami et al., 2005). KA413 [dnaA46(Ts)] cells were transformed with $250 \mathrm{ng}$ plasmid bearing the indicated $d n a A$ allele and incubated on LB agar containing thymine $(50 \mu \mathrm{g} / \mathrm{ml})$ and spectinomycin $(100 \mu \mathrm{g} / \mathrm{mL})$ at $30^{\circ} \mathrm{C}$ or plated on $\mathrm{LB}$ agar containing the same reagents as well as $10 \mathrm{mM}$ arabinose at $42^{\circ} \mathrm{C}$. CFU were calculated to determine the transformation efficiency.

\section{Limited Trypsin Digestion Assay}

Trypsin cleavage of DnaA was performed as described (Mizushima et al., 1998). DnaA protein was pre-incubated with $2 \mathrm{mM}$ ATP or ADP at $0^{\circ} \mathrm{C}$ for $15 \mathrm{~min}$ and further incubated with $160 \mathrm{ng}$ of trypsin in buffer [50 mM Tricine- $\mathrm{KOH}(\mathrm{pH} 8.25)$, $0.5 \mathrm{mM}$ magnesium acetate, $0.3 \mathrm{mM}$ EDTA, $7 \mathrm{mM}$ dithiothreitol, $20 \%$ (v/v) glycerol, and $0.007 \%$ Triton $\mathrm{X}-100$ ] at $30^{\circ} \mathrm{C}$ for $30 \mathrm{~min}$. The reaction was terminated by addition of SDS-sample buffer and samples were determined by Western blot analysis.

\section{Electrophoretic Mobility Shift Assay (EMSA)}

Experiments using a minimal oriC-containing fragment were performed essentially as previously described (Kawakami et al., 2005). The FAM labeled 469 bp oriC was prepared by PCR with the primers $5^{\prime}$ FAM-oriC $\mathrm{F}$ and $5^{\prime}$ FAM-oriC $\mathrm{R}$. The indicated amounts of DnaA protein were incubated for $5 \mathrm{~min}$ at $20^{\circ} \mathrm{C}$ in buffer containing $20 \mathrm{mM}$ HEPES-KOH, $\mathrm{pH} 8.0$, $5 \mathrm{mM}$ magnesium acetate, $1 \mathrm{mM}$ EDTA, $4 \mathrm{mM}$ dithiothreitol, $0.2 \%$ Triton X-100, $5 \%(\mathrm{v} / \mathrm{v})$ glycerol, $0.5 \mathrm{mg} / \mathrm{ml} \mathrm{BSA}$, the oriC fragment $(0.13 \mathrm{pmol})$ and $2 \mathrm{mM}$ ATP. Reaction products were analyzed by $5 \%$ PAGE in cold $0.5 x$ TBE buffer $(44.5 \mathrm{mM}$ Tris, $44.5 \mathrm{mM}$ boric acid, $1 \mathrm{mM}$ EDTA) and detected by FUJIFILM FLA7000. Experiments using $d n a A$ promoter amplified by 
$5^{\prime}$ FAM-PdnaA $\mathrm{F}$ and $5^{\prime}$ FAM-PdnaA $\mathrm{R}$ were performed in the same way.

Experiments using DARS1 were performed according to published methods (Fujimitsu et al., 2009). DARS1 was amplified using $5^{\prime}$ FAM-DARS1 $\mathrm{F}$ and $5^{\prime}$ FAM-DARS1 R, ADP-DnaA was prepared by incubation of DnaA with $2 \mu \mathrm{M}$ ADP for $15 \mathrm{~min}$ at $0^{\circ} \mathrm{C}$. ADP-DnaA was incubated for $5 \mathrm{~min}$ at $30^{\circ} \mathrm{C}$ in $12.5 \mu \mathrm{l}$ of buffer [20 mM HEPES-KOH at $\mathrm{pH} 7.6,10 \mathrm{mM}$ magnesium acetate, $1 \mathrm{mM}$ EDTA, $8 \mathrm{mM}$ dithiothreitol, $0.1 \mathrm{mg} / \mathrm{ml}$ bovine serum albumin, $5 \%$ glycerol, $50 \mathrm{mM}$ potassium glutamate, $2 \mathrm{mM}$ ADP, $21 \mathrm{ng}$ poly(dI-dC), $100 \mathrm{nmol}$ of DARS1].

\section{In Vitro Modification Assay}

All in vitro modification assays were performed as described (Ren et al., 2016; Zhang et al., 2016). For YfiQ modification assay, DnaA was incubated at $37^{\circ} \mathrm{C}$ for $6 \mathrm{~h}$ in the presence or absence of YfiQ as well as Ac-CoA. For CobB modification assay, DnaA protein was incubated at $30^{\circ} \mathrm{C}$ for $6 \mathrm{~h}$ in the presence or absence of CobB as well as $\mathrm{NAD}^{+}$. For AcP modification assay, DnaA was incubated at $37^{\circ} \mathrm{C}$ in the in the presence or absence of $20 \mathrm{mM}$ AcP, samples were collected at indicated time.

\section{DNase I Footprint Assay}

For preparation of fluorescent FAM labeled probes, the promoter region of oriC was PCR amplified with Dpx DNA polymerase (TOLO Biotech) from the plasmid pUC18B-T oriC using primers of M13F-47(FAM) and M13R-48. The FAM-labeled probes were purified by the Wizard SV Gel and PCR Clean-Up System (Promega) and were quantified with NanoDrop 2000C (Thermo Scientific).

DNase I footprint assays were performed similar to Wang et al. (2012). For each assay, $700 \mathrm{ng}$ probes were incubated with different amounts of WT and K243Ac in a total volume of $40 \mu \mathrm{l}$. After incubation for $30 \mathrm{~min}$ at $25^{\circ} \mathrm{C}, 10 \mu \mathrm{l}$ solution containing about $0.015 \mathrm{u}$ DNase I (Promega) and $100 \mathrm{nmol}$ freshly prepared $\mathrm{CaCl}_{2}$ was added and further incubated for $1 \mathrm{~min}$ at $25^{\circ} \mathrm{C}$. The reaction was stopped by adding $140 \mu \mathrm{l}$ DNase I stop solution (200 mM unbuffered sodium acetate, $30 \mathrm{mM}$ EDTA and 0.15\% SDS). Samples were firstly extracted with phenol/chloroform, then precipitated with ethanol and the pellets were dissolved in $30 \mu \mathrm{l}$ Milli $\mathrm{Q}$ water. The preparation of the DNA ladder, electrophoresis and data analysis were the same as described before (Wang et al., 2012), except that the GeneScan-LIZ500 size standard (Applied Biosystems) was used.

\section{Western Blot Analysis}

Briefly, DnaA protein samples were separated by $10 \%$ SDS-PAGE, transferred to PVDF membranes. For acetylation Western blot, $50 \mathrm{mM}$ Tris- $\mathrm{HCl}$ (pH 7.5) with100 mM NaCl, 10\% (V/V) Tween20 and $1 \%$ peptone (Amresco) was used for blocking. $50 \mathrm{mM}$ Tris- $\mathrm{HCl}(\mathrm{pH} 7.5)$ with $150 \mathrm{mM} \mathrm{NaCl}, 0.5 \%$ (V/V) Tween20 and 5\% non-fat milk was used for anti-DnaA and anti-His tag Western blot. The rabbit anti-sera against DnaA (1:5000) or anti-AcK (1:1000) or anti-DnaA K243AcK (1:1000) and the mouse anti-sera against-His (1:4000) were used as the primary antibodies and incubated with the membranes overnight at $4^{\circ} \mathrm{C}$. Goat horseradish peroxidase (HRP)-conjugated anti-rabbit or anti-mouse IgG antibodies (1: 4000) were used as the secondary antibodies and incubated at room temperature for about $1 \mathrm{~h}$. Blots were scanned with G: BOX Chemi system (Syngene) and relative gray value was quantified by Image $\mathrm{J}$.

\section{Site-directed Mutagenesis of dnaA}

Base substitutions were introduced into the wild-type dnaA allele by corresponding primers using KOD-Plus-Mutagenesis Kit according to the manufacturer's instructions (Toyobo, SMK-101). All the site-directed mutants were confirmed by DNA sequencing.

\section{Identification of Acetylated Lysine Residues by Mass Spectrometry}

The purified chromosomally encoded DnaA was separated by $10 \%$ one-dimensional SDS-PAGE and the bands containing DnaA were excised. The excised bands were destained and dehydrated. For trypsin digestion, proteins were treated with $100 \mathrm{mM}$ DTT at $56^{\circ} \mathrm{C}$ for $30 \mathrm{~min}$ and then treated with $100 \mathrm{mM} \mathrm{NH}_{4} \mathrm{HCO}_{3}$ at room temperature for $15 \mathrm{~min}$. The freeze-dried samples were incubated with 100-200 ng trypsin at $37^{\circ} \mathrm{C}$ for $20 \mathrm{~h}$. Peptides generated after proteolytic digestion of DnaA were separated by the EASY-nLC HPLC system (Thermo Scientific) and analyzed by Q-Exactive mass spectrometer (Thermo Scientific). Mass spectrometric data were analyzed using the Mascot 2.2 software for database search.

\section{The Anti-DnaA K243Ac Specific Polyclonal Antibody Preparation}

The peptide AC-CQFFANK(Ac)ERS- $\mathrm{NH}_{2}$ conjugated to BSA was used as immune peptide to immunize rabbits. During 2 months, rabbits were immunized for six times, and the antiserum was collected, and control peptide ACCQFFANKERS-NH $\mathrm{N}_{2}$ was used to remove non-specific antibody. The sensitivity and specificity of antibody were evaluated by ELISA and Western blot.

\section{RESULTS}

\section{DnaA K243 Is Required for In Vivo DNA Replication Initiation}

Our previous work showed that DnaA is acetylated and that its acetylation level changes at different growth stages in E. coli (Zhang et al., 2016). Here, we purified natively expressed DnaA and identified another acetylated residue, K243 (Figure 1A), by mass spectrometry. DnaA is ubiquitous in bacteria (Skarstad and Boye, 1994), and a sequence alignment showed that K243 is highly conserved in eubacteria and archaea (Figure 1B). K243 is located between helices $\alpha 5$ and $\alpha 6$ of DnaA, suggesting that its acetylation may affect single-stranded DNA binding by DnaA (Duderstadt et al., 2011). To evaluate the role of K243 acetylation in vivo, we performed a plasmid complementation test using E. coli strain KA413 (Kawakami et al., 2005), which contains a temperature-sensitive DnaA46 protein that is unstable at $42^{\circ} \mathrm{C}$. K243 was mutated either to glutamine (Q), to mimic an acetylated 

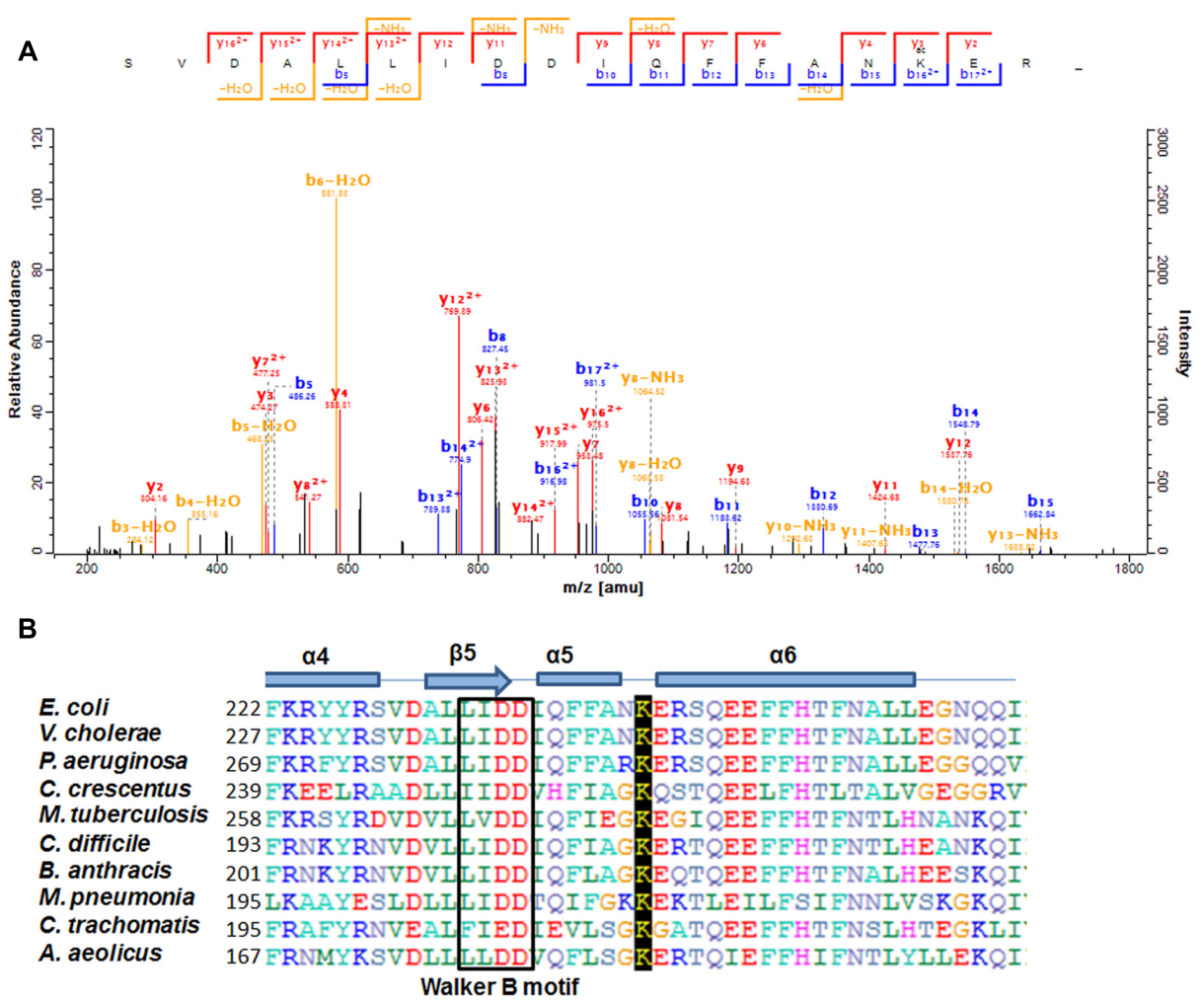

C

\begin{tabular}{|c|c|c|c|c|}
\hline \multirow{2}{*}{ Plasmid } & \multirow{2}{*}{ Allele } & \multicolumn{3}{|c|}{ 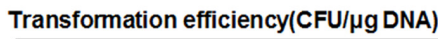 } \\
\hline & & $30^{\circ} \mathrm{C}$ & $42^{\circ} \mathrm{C}$ & $42^{\circ} \mathrm{C} / 30^{\circ} \mathrm{C}$ \\
\hline pCDSS-K & WT & $4.80^{\star} 10^{6}$ & $5.60^{*} 10^{6}$ & 1.17 \\
\hline pCDSS-Q & K243Q & $7.96^{*} 10^{6}$ & 41 & $5.15^{\star} 10^{-6}$ \\
\hline pCDSS-R & K243R & $8.48^{*} 10^{6}$ & $4.32^{*} 10^{6}$ & 0.51 \\
\hline pCDSs & None & $6.40^{*} 10^{6}$ & 0 & 0 \\
\hline
\end{tabular}

D

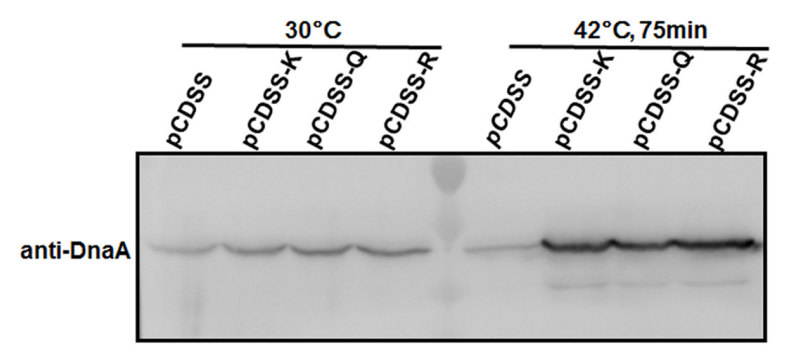

FIGURE 1 | DnaA K243 is required for in vivo DNA replication initiation. (A) Acetylation of DnaA K243 identified by mass spectrometry. Native DnaA protein was purified and analyzed by liquid chromatography-tandem mass spectrometry after chymotrypsin digestion. Shown is the spectrum covering the region from 200 to $1800 \mathrm{~m} / \mathrm{z}$, which includes the peptide containing the acetylated K243 residue. (B) Alignment of DnaA protein sequences. Comparison of E. coli DnaA from F222 to 1264 with the corresponding DnaA regions from other strains using a Clustal W multiple alignment. Secondary structures are also shown (helices $\alpha 4-6$, sheet $\beta 5$, and the Walker B motif). E. coli, Escherichia coli; V. cholerae, Vibrio cholerae; P. aeruginosa, Pseudomonas aeruginosa; C. crescentus, Caulobacter crescentus; M. tuberculosis, Mycobacterium tuberculosis; C. difficile, Clostridium difficile; B. anthracis, Bacillus anthracis; M. pneumoniae, Mycoplasma pneumoniae; C. trachomatis; Chlamydia trachomatis; A. aeolicus; Aquifex aeolicus. (C) Plasmid complementation assay. KA413 [dnaA46 (Ts)] transformed with the indicated plasmids was incubated on LB agar (containing $50 \mu \mathrm{g} / \mathrm{mL}$ thymine and $100 \mu \mathrm{g} / \mathrm{mL}$ spectinomycin) at $30^{\circ} \mathrm{C}$, and on LB agar containing the same reagents in addition to $10 \mathrm{mM}$ arabinose at $42^{\circ} \mathrm{C}$. Colony-forming units (CFU) were calculated to determine the transformation efficiency and the ratios of $\mathrm{CFU}$ at $42^{\circ} \mathrm{C} / 30^{\circ} \mathrm{C}$ are shown. (D) Western blot analysis of DnaA. KA413 cells (dnaA46) bearing pCDSS (empty vector), pCDSS-K (vector expressing wild-type dnaA), pCDSS-Q (vector expressing the $\mathrm{K} 243 \mathrm{Q}$ mutant), or pCDSS-R (vector expressing the $\mathrm{K} 243 \mathrm{R}$ mutant) were grown at $30^{\circ} \mathrm{C}$ in $\mathrm{LB}$ medium containing thymine $(50 \mu \mathrm{g} / \mathrm{mL})$, spectinomycin $(100 \mu \mathrm{g} / \mathrm{mL})$, and arabinose $(10 \mathrm{mM})$ until the optical density at $600 \mathrm{~nm}\left(\mathrm{OD}_{600}\right)$ reached 0.2. A portion $(5 \mathrm{ml})$ of each culture was withdrawn, and the remainder was further incubated for $75 \mathrm{~min}$ at $42^{\circ} \mathrm{C}$, at which time an additional portion (approximately $1 \mathrm{ml}$ ) was withdrawn. Proteins in each sample were subjected to $10 \%$ sodium dodecyl sulfate-polyacrylamide gel electrophoresis (SDS-PAGE), and a western blot analysis was performed using an anti-DnaA antibody.

lysine, or arginine (R), to prevent acetylation but retain the positive charge (Ren and Gorovsky, 2001). The plasmids bearing the wild-type or mutant $d n a A$ alleles were introduced into $E$. coli strain KA413, colonies of transformants formed with a similar efficiency at $30^{\circ} \mathrm{C}$. However, when transformants were incubated at $42^{\circ} \mathrm{C}$, the plasmid bearing the dnaA K243Q mutation did not support colony formation, while plasmids bearing wild-type $d n a A$ or the dnaA K243R mutant grew well (Figure 1C). To exclude the possibilities of low expression or instability of DnaA $\mathrm{K} 243 \mathrm{Q}$, we determined the DnaA levels in these strains. Western 
blot analysis showed that the DnaA levels were comparable in the different strains at both temperatures (Figure 1D). These results demonstrate that $\mathrm{K} 243$ is required for DnaA activity in vivo, and suggest that K243 acetylation may be involved in the regulation of DnaA activity.

\section{Acetylation of K243 Does Not Affect the ATP/ADP Binding Ability of DnaA}

To test whether K243 acetylation plays an important role in DnaA activity, we produced a homogeneous, recombinant protein (Supplementary Figure S1) that contains $\mathrm{N}^{\varepsilon}$-acetyllysine at K243 (DnaA K243Ac) using a previously described strategy (Neumann et al., 2008). With high translational fidelity and efficiency, this system has been used widely to decipher the role of acetylation in biological systems (Arbely et al., 2011; Ren et al., 2016; Zhang et al., 2016). Western blot analysis indicated that the acetylation level of K243Ac was much higher than that of the wild-type DnaA (Figure 2A).

We have demonstrated that acetylation of the conserved K178 suppresses its binding to ATP/ADP (Zhang et al., 2016). Because K243 is located in the same domain of DnaA as K178, and ATP/ADP binding activity is critical for the function of DnaA, we assessed the binding affinities of DnaA K243Ac using a limited trypsin digestion assay (Mizushima et al., 1998). In the absence of ATP or ADP, DnaA was digested completely by trypsin; however, in the presence of $2 \mathrm{mM}$ ATP or ADP, limited trypsinolysis of the wild-type DnaA and DnaA K243Ac both produced a predominant $30-\mathrm{kDa}$ peptide (Figure $2 \mathrm{~B}$ ), which indicates that their binding affinities for ATP and ADP are comparable. These results suggest that $\mathrm{K} 243$ is not required for ATP and ADP binding, which is consistent with a previous study demonstrating that a K243A mutation does not affect the ATP/ADP binding affinity of DnaA (Ozaki et al., 2008).

\section{Acetylation of K243 Inhibits the Formation of the oriC/DnaA Complex}

DnaA interacts strongly with DNA. As an initiator, DnaA binds to the replication origin of $E$. coli, resulting in unwinding of an adjacent AT-rich region. In addition to its primary function as the initiator, DnaA also acts as a transcription factor that represses or activates the expression of several genes, depending on the location and arrangement of their DnaA boxes (Messer and Weigel, 1997). We analyzed complexes formed by oriC and DnaA using an electrophoretic mobility shift assay (EMSA) (Kawakami et al., 2005) (Figure 3A). The results showed that wild-type DnaA formed multimeric complexes with oriC DNA in a manner dependent on the amount of DnaA. When DnaA K243Ac was used, we also observed the formation of homomultimers, but more K243Ac was required to form the similar complex pattern, compared with wild-type DnaA. This result indicates that DnaA K243Ac showed a decreased ability in binding oriC.

DnaA can auto-regulate its own expression (Braun et al., 1985); thus, we determined its $d n a A$ promoter-binding activity in the same way. The results showed that wild-type DnaA and DnaA K243Ac had similar dnaA promoter-binding abilities (Figure 3B). ADP-DnaA can also bind the DARS to regenerate ATP-DnaA via nucleotide exchange (Fujimitsu et al., 2009). Thus, we assessed the binding kinetics of DnaA K243Ac to DARS1 (Supplementary Figure S2), and we found that, similar to the wild-type DnaA, DnaA K243Ac formed complexes with DARS1. These results indicate that $\mathrm{K} 243 \mathrm{Ac}$ retains the ability to bind other DNA sequences, except oriC.

Because K243Ac showed decreased oriC binding activity, we speculate that this mutation may affect the higher-order structure of the oriC/DnaA multimer complex. Therefore, a DNase I footprinting assay (Wang et al., 2012) was employed to assess the formation of the ATP-DnaA-specific complex on oriC. Similar to the wild-type ATP-DnaA, K243Ac bound to boxes R1, R2, R3, R4, $\mathrm{M}, \mathrm{I} 1$, and $\mathrm{C} 2$, but it failed to recognize the low-affinity boxes I3, C1, and C3 (Figure 3C). Additionally, we identified a novel lowaffinity site that was bound by the wild-type ATP-DnaA. This site has the sequence $5^{\prime}$-TTAAGATCA-3', and it is located between boxes $\mathrm{R} 1$ and $\tau 1$. These results suggest that DnaA K243Ac is defective in forming a complete initiation complex structure, indicating that residue $\mathrm{K} 243$ is necessary for the formation of the intact complex.

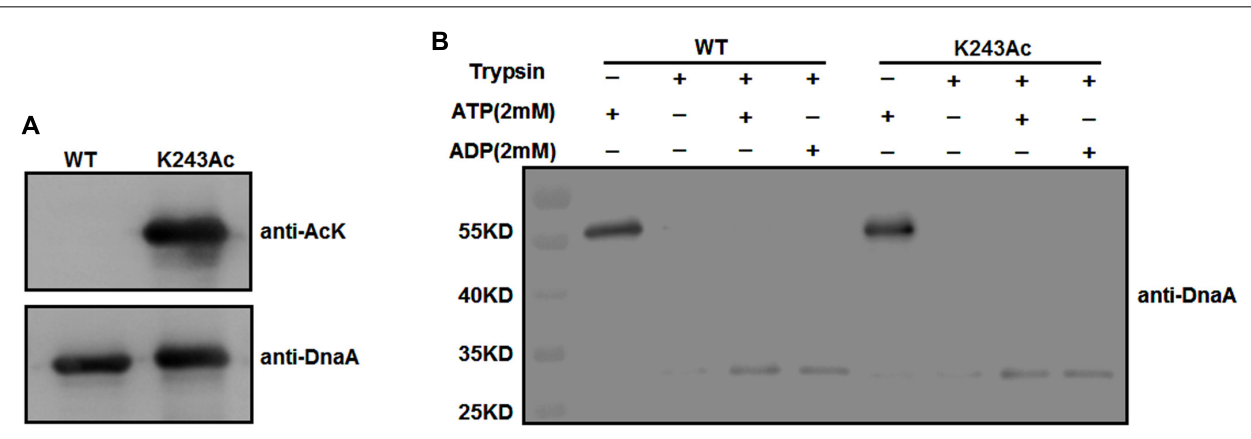

FIGURE 2 | Purification and ATP/ADP binding activity of DnaA K243Ac. (A) Immunoblot analysis of DnaA K243Ac. K243Ac was produced by a site-specific acetylation system in the presence of pAcKRS-3 and pCDF-PylT-dnaA (K243TAG). Proteins (purity > 90\%) were probed with an anti-acetylated-lysine antibody, and anti-DnaA was used as a loading control. (B) ATP/ADP binding activity of DnaA K243Ac. DnaA proteins were preincubated with 2 mM ATP or ADP at $0^{\circ} \mathrm{C}$ for $15 \mathrm{~min}$ and further incubated with $160 \mathrm{ng}$ of trypsin at $30^{\circ} \mathrm{C}$ for $30 \mathrm{~min}$. The reaction was terminated, and samples were analyzed by Western blot using an anti-DnaA antibody. 

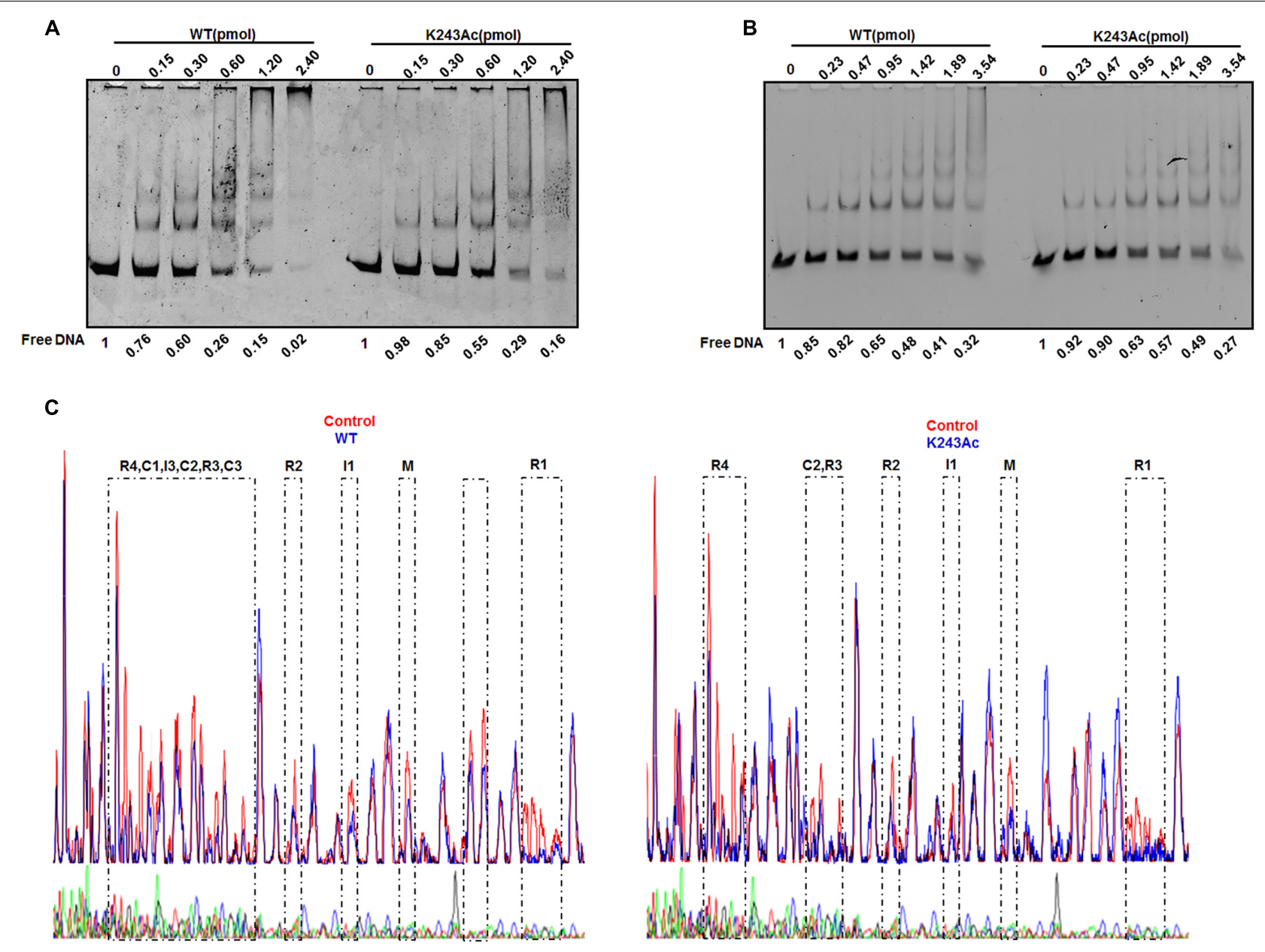

FIGURE 3 | DNA binding activity of DnaA K243Ac. (A) oriC binding activities assessed by EMSA. Various amounts (0-2.4 pmol) of the wild- type and DnaA K243Ac proteins were incubated with FAM-labeled oriC for 5 min at $20^{\circ} \mathrm{C}$. Reaction products were analyzed by $5 \%$ PAGE and detected using an FLA-7000 image analysis system (Fujifilm, Tokyo, Japan). Free DNA was quantified by Image J. (B) The dnaA promoter binding activity was determined by EMSA, similar to that used for the oriC binding assay, except different DNAs were used. Free DNA was quantified by Image J. (C) DNase I footprinting assay. Seven hundred nanograms of FAM-labeled probes were incubated with wild-type DnaA or DnaA K243Ac. After incubation for 30 min at $25^{\circ} \mathrm{C}$, approximately 0.015 units of DNase I (Promega, Madison, WI, USA) and $100 \mathrm{nmol}$ of freshly prepared $\mathrm{CaCl}_{2}$ were added and further incubated for 1 min at $25^{\circ} \mathrm{C}$. The reaction was terminated by adding stop buffer, and the DNA was purified. Electrophoresis and data analysis were performed as described previously (Wang et al., 2012). No protein control and the WT and K243Ac mutant protein profiles were overlaid. The DnaA-binding regions are indicated.

\section{The Acetylation Level of K243 Is Regulated by CobB and Acetyl Phosphate (AcP)}

To examine the relationship between K243 acetylation and DnaA activity in more detail, it is of interest to identify the factors responsible for regulating the acetylation level of DnaA K243. To do so, we first prepared a K243 site-specific acetylation antibody and confirmed its specificity and sensitivity by Western blot (Supplementary Figure S3). Although E. coli contains multiple genes that encode putative acetyltransferases, YfiQ is the only acetyltransferase identified thus far (Starai and EscalanteSemerena, 2004; Liang and Deutscher, 2012), and our previous work demonstrated that YfiQ can acetylate DnaA (Zhang et al., 2016). To determine whether YfiQ can acetylate DnaA K243 directly, the wild-type DnaA was incubated with purified YfiQ and the acetyl group donor acetyl-CoA (Ac-CoA). As shown in Figure 4A, the acetylation level of K243 increased significantly, which means that $\mathrm{K} 243$ is a substrate of YfiQ in vitro. In addition to YfiQ, a non-enzymatic mechanism of acetylation exists, which is dependent on AcP (Weinert et al., 2013; Kuhn et al., 2014). We next examined the involvement of AcP in the acetylation of DnaA K243. Increased acetylation was observed in a timedependent manner, indicating that AcP can acetylate DnaA K243 in vitro (Figure 4B). Acetylation can be a reversible and dynamic process that is reversed enzymatically by lysine deacetylase (Starai et al., 2002). As the predominant deacetylase in E. coli, CobB plays a major role in the deacetylation of many substrates (Starai et al., 2002; AbouElfetouh et al., 2015). Thus, we purified the K243Ac protein with a high acetylation level, and treated it with $\mathrm{CobB}$ in the presence of $\mathrm{NAD}^{+}$. The results showed that 
A

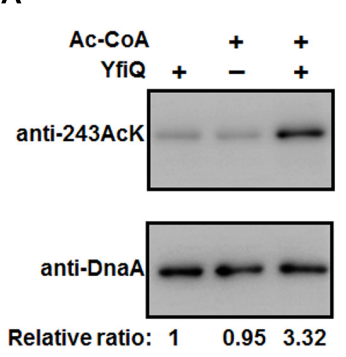

B
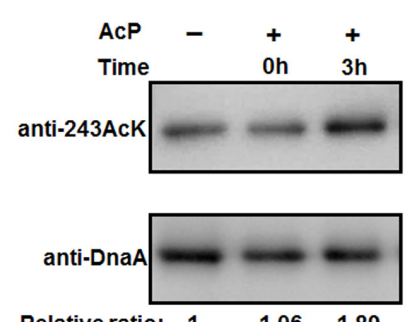

C
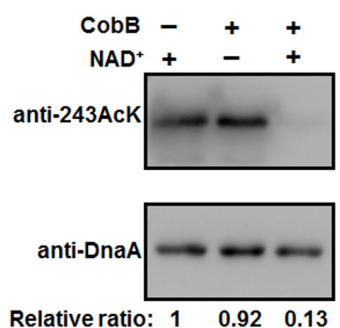

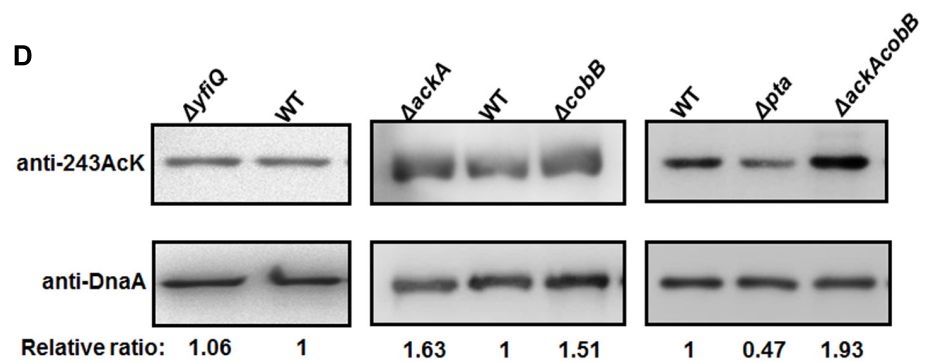

E
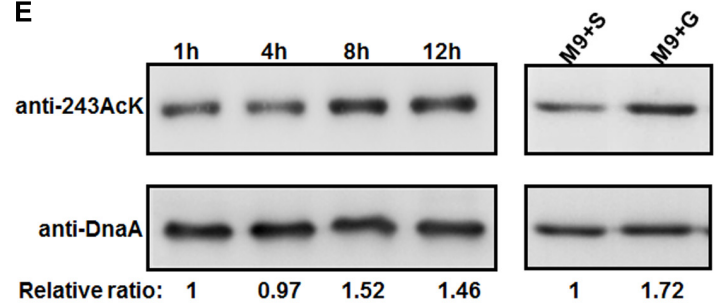

FIGURE 4 | The acetylation of K243 is regulated by CobB and AcP. (A) In vitro YfiQ modification assay. Wild-type DnaA and DnaA K243Ac were incubated in the presence or absence of $\mathrm{YfiQ}$, as well as the cofactor Ac-CoA. (B) In vitro AcP modification assay. Wild-type DnaA and DnaA K243Ac were incubated at $37^{\circ} \mathrm{C}$ in the presence or absence of $20 \mathrm{mM} \mathrm{AcP}$, and samples were collected at the indicated times. (C) In vitro CobB deacetylation assay. The mutant DnaA K243Ac protein was treated with CobB in the presence or absence of $\mathrm{NAD}^{+}$. (D) CobB and AcP can regulate the acetylation level of K243 in vivo. DnaA protein from the wild-type strain and the indicated deletion strains were cultured in LB medium to the mid-exponential phase and purified. (E) The acetylation level of K243 correlates with the intracellular AcP concentration. Cells were grown overnight and then diluted into fresh LB medium supplemented with $0.4 \%$ glucose to an $\mathrm{OD}_{600}$ of 0.1 . Then, the cells were collected at different time points to purify DnaA. Cells grown in M9 minimal medium supplemented with $0.4 \%$ glucose (M9+G) or $0.4 \%$ succinate (M9+S) to the early stationary phase were also collected to purify DnaA. In Figure 4, all the DnaA proteins were resolved on 10\% SDS-PAGE and probed with anti-DnaA and anti-DnaA (K243AcK) antibodies. The relative ratios are referred to as the anti-K243Ack: anti-DnaA ratios. Western blots are representative from at least three independent replicates.

the acetylation level of K243 decreased significantly after CobB treatment (Figure 4C).

We also determined the acetylation level of K243 in vivo by purifying native DnaA during the mid-exponential phase from different strains (Figure 4D). We found that the acetylation level of K243 in the $y f i Q$ mutant was comparable to that in the wildtype strain, which means that YfiQ cannot acetylate K243 in vivo. However, in an ack $A$ deletion strain, which can accumulate a high level of intracellular AcP (Weinert et al., 2013; Kuhn et al., 2014), the acetylation of DnaA K243 was higher than that of DnaA from the wild-type strain. When the deacetylase CobB was absent, the acetylation of DnaA K243 also increased significantly. These in vivo assays were repeated in an E. coli strain MG1655
(Supplementary Figure S4), and the results were the same as those in E. coli strain BL21. Additionally, the acetylation level of K243 was higher in the ack $A$ and $\operatorname{cobB}$ double deletion mutant than in the single deletion mutants (Figure 4D).

To complete the epistasis analysis, we also assessed the acetylation level of K243 in a pta mutant. The acetylation level of K243 decreased by approximately 50\%, but it was still detectable in the pta deletion strain (Figure 4D), which cannot produce AcP (Weinert et al., 2013; Kuhn et al., 2014), suggesting the existence of an AcP-independent acetylation mechanism. Taken together, we conclude that the in vivo acetylation level of K243 depends on AcP, CobB, and a yet unknown factor, but not YfiQ, although YfiQ could acetylate DnaA on other lysine residues (Zhang et al., 


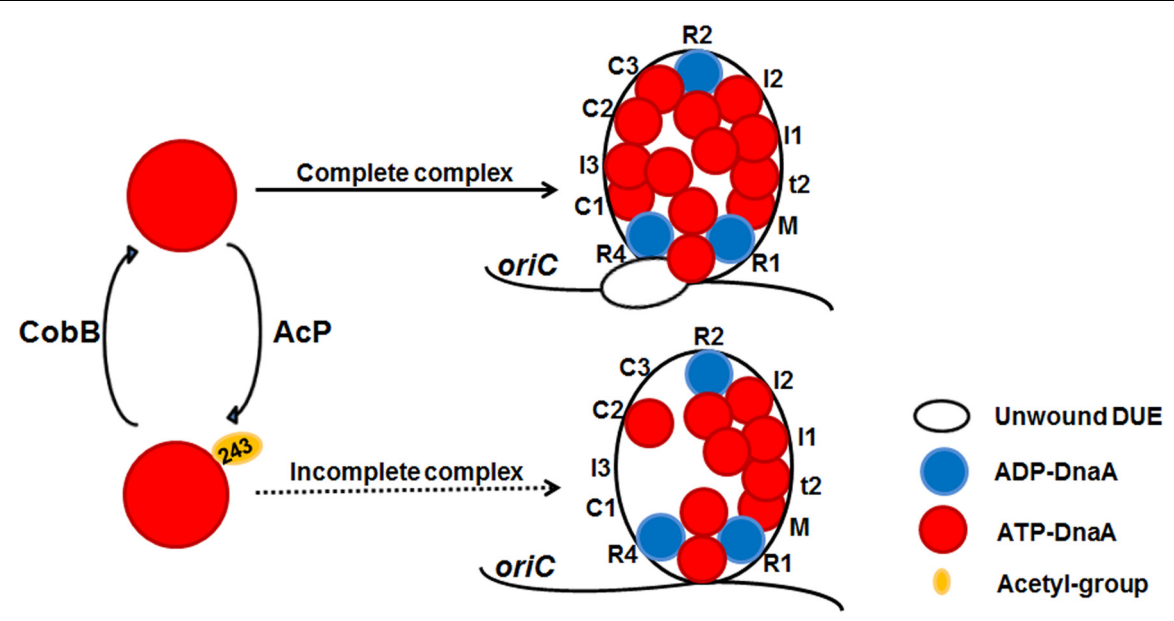

FIGURE 5 | A model of reversible acetylation of DnaA K243 regulating DNA replication initiation. AcP and CobB are responsible for regulating the dynamic acetylation level of DnaA K243 in vivo. When the acetylation level of DnaA K243 is low, ATP-DnaA can bind to all the DnaA boxes within oriC. The formation of a complete initiation complex triggers DUE unwinding. When the acetylation level of DnaA K243 is high, ATP-DnaA fails to bind to several low-affinity DnaA boxes. This incomplete oriC-DnaA complex has defect in promoting DUE unwinding.

2016). To determine a more precise relationship between the intracellular AcP and acetylation level of K243, we grew the wildtype cells (E. coli strain BW25113) in LB broth supplemented with $0.4 \%$ glucose, and we harvested the cells at different time points. The signal intensity of K243 acetylation levels increased at 8 and $12 \mathrm{~h}$ (Figure $4 \mathrm{E}$ ), which is consistent with a previous report demonstrating that the glucose-induced global acetylation profile increased significantly after entry into stationary phase, at which time AcP begins to accumulate (Schilling et al., 2015). Additionally, cells have a larger acetyl-CoA pool if the carbon source is glucose, rather than succinate (Chohnan et al., 1998); thus, we speculate that the AcP level is higher in a glucosecontaining medium. As expected, the acetylation level of K243 was higher when cells were grown in M9 minimal medium supplemented with glucose $(\mathrm{M} 9+\mathrm{G})$, compared with that in a succinate-supplemented medium $(\mathrm{M} 9+\mathrm{S})$ (Figure 4E). These results demonstrate that the acetylation level of K243 correlates with the intracellular AcP concentration.

\section{DISCUSSION}

Like other ATPases associated with diverse cellular activities, bacterial DnaA has a functional conformation that consists of spiral or open-ring-shaped high-order oligomers with a central pore (Ozaki et al., 2008). Certain residues within the pore surface interact directly with oriC and play crucial roles in specific DnaA activities. E. coli DnaA K243 is located exactly in the central cavity (Ozaki et al., 2008), and we identified its acetylation by mass spectrometry. Our previous study proposed a model in which protein acetylation controls DNA replication initiation by targeting DnaA at the key residue K178 in the Walker A motif. A K178Ac mutant lacks the ability to bind ATP and ADP, and, therefore, it cannot initiate DNA replication (Zhang et al., 2016). In this study, we proposed a working model to display the role of acetylation of DnaA K243 in DNA replication initiation process (Figure 5). As a conserved lysine residue located in the same domain of DnaA (Figure 1A), the acetylation of K243 did not lower the affinity of DnaA for ATP/ADP (Figure 2B), suggesting that $\mathrm{K} 243$ acetylation regulates DnaA activity via a different mechanism. EMSA assays showed that DnaA K243Ac had a slightly impaired binding to oriC (Figure 3A), but it bound similarly to other DNAs, such as the dnaA promoter and DARS1, compared to wild-type DnaA (Figure 3B and Supplementary Figure S2). DNase I footprinting assay revealed that K243Ac cannot recognize several low-affinity boxes, including $\mathrm{C} 1, \mathrm{C} 2$, and I3 (Figure 3C). In addition, our DNase I footprinting assay identified a novel site that is bound by the wild-type DnaA, but not by DnaA K243Ac. The novel site is positioned between boxes $\mathrm{R} 1$ and $\tau 1$, and its sequence is $5^{\prime}$-TTAAGATCA- $3^{\prime}$; its role in DNA replication will be investigated in future studies.

Ozaki et al. (2008) reported that a K243A mutant was incapable of binding to ATP-DnaA-specific sites such as the box $\mathrm{M}$ and $\mathrm{I} / \tau$-sites. This inconsistency is mainly due to the different DnaA mutants used. In our view, K243Ac more accurately represents the true physiological state of the protein, whereas the K243A mutant lacks the lysine side chain completely, which might fully abrogate the function of this residue. Our DNase I footprinting assay did not detect the binding of ATP-DnaA to the box in the 13-mer region, or to the I2 box and $\tau$ sites. This difference may be caused by the different DNase I footprinting method that we used, or the DnaA purification methods. ADPDnaA is unable to form an open complex (Sekimizu et al., 1987; Crooke et al., 1992), although it binds to oriC $\mathrm{R}$ boxes with the same affinity as ATP-DnaA; therefore, we did not examine the binding activity of ADP-DnaA.

Either the diminished positive charge or the side chain structure of $\mathrm{K} 243 \mathrm{Ac}$ inhibits its interaction with the oriC region (Wagner and Payne, 2011), especially with low-affinity DnaA binding sites. All these low-affinity DnaA binding sites are 
required to form replication-efficient pre-replication complexes, and, therefore, they are instrumental for oriC unwinding (McGarry et al., 2004; Rozgaja et al., 2011). In addition, the I3 site acts as an important determinant of ATP-DnaA-dependent oriC separation, and mutation of a specific position of the I3 site lost its discrimination for ATP-DnaA allowing ADP-DnaA binding (McGarry et al., 2004). Every DnaA box in oriC has a function in the initiation process (Langer et al., 1996), therefore, we speculate that the incomplete complex formed by DnaA K243Ac with oriC could cause a DNA replication defect.

Lysine acetylation is an abundant post-translational modification in bacteria, and it is involved in a broad range of cellular events such as central metabolism (Wang et al., 2010), transcription (Qin et al., 2016), virulence (Starai and EscalanteSemerena, 2004; Sang et al., 2016), and stress responses (Ma and Wood, 2011). Acetylation is catalyzed by the Gcn5-like acetyltransferase YfiQ (Starai and Escalante-Semerena, 2004) and chemically by AcP (Weinert et al., 2013; Kuhn et al., 2014) in E. coli. The $\mathrm{NAD}^{+}$dependent Sir2 homolog CobB (Starai et al., 2002) and the newly found deacetylase YcgC (Tu et al., 2015) can reverse a fraction of the acetylated lysine residues. However, we did not account for YcgC, considering its relatively low expression level (unpublished data). We found that YfiQ can modify DnaA $\mathrm{K} 243$ in vitro (Figure 4A) but not in vivo (Figure 4D), although it acetylates DnaA at K178. We speculate that purified DnaA may have a different conformation than the native protein in vivo, and that YfiQ can access DnaA K243 in vitro, but not in vivo. Alternatively, the specificity of YfiQ is low and its in vivo concentration was not sufficient for DnaA acetylation on K243. Non-enzymatic acetylation with AcP is more global (Weinert et al., 2013; Kuhn et al., 2014; Schilling et al., 2015) compared with YfiQ-catalyzed acetylation, and this small molecule can acetylate DnaA K243 both in vitro (Figure 4B) and in vivo (Figure 4D). The deacetylase CobB can effectively remove the acetyl group of DnaA K243 (Figures 4C,D), thus, bacteria can dynamically adjust the acetylation level of DnaA K243 in response to environmental stimuli. The detectable acetylation level of K243 in

\section{REFERENCES}

AbouElfetouh, A., Kuhn, M. L., Hu, L. I., Scholle, M. D., Sorensen, D. J., Sahu, A. K., et al. (2015). The E. coli sirtuin CobB shows no preference for enzymatic and nonenzymatic lysine acetylation substrate sites. Microbiologyopen 4, 66-83. doi: $10.1002 / \mathrm{mbo} 3.223$

Arbely, E., Natan, E., Brandt, T., Allen, M. D., Veprintsev, D. B., Robinson, C. V., et al. (2011). Acetylation of lysine 120 of p53 endows DNA-binding specificity at effective physiological salt concentration. Proc. Natl. Acad. Sci. U.S.A. 108, 8251-8256. doi: 10.1073/pnas.1105028108

Baker, T. A., and Bell, S. P. (1998). Polymerases and the replisome: machines within machines. Cell 92, 295-305. doi: 10.1016/S0092-8674(00)80923-X

Braun, R. E., O'Day, K., and Wright, A. (1985). Autoregulation of the DNA replication gene dnaA in E. coli K-12. Cell 40, 159-169.

Chohnan, S., Izawa, H., Nishihara, H., and Takamura, Y. (1998). Changes in size of intracellular pools of coenzyme A and its thioesters in Escherichia coli K-12 cells to various carbon sources and stresses. Biosci. Biotechnol. Biochem. 62, 1122-1128. doi: 10.1271/bbb.62.1122

Crooke, E., Castuma, C. E., and Kornberg, A. (1992). The chromosome origin of Escherichia coli stabilizes DnaA protein during rejuvenation by phospholipids. J. Biol. Chem. 267, 16779-16782. the pta mutant strain (Figure 4D) demonstrates conclusively that AcP-independent factors are involved in regulating acetylation level of K243. Additionally, we cannot rule out the possibility that another deacetylation pathway exists in addition to CobB. Approximately $70 \%$ of the lysine residues of DnaA were identified as substrates for acetylation in E. coli, and it is very likely that other acetylated lysine residues have an impact on the activity of DnaA. We believe this reversible, dynamic modification is an efficient way to coordinate the initiation process with environmental changes, which should not be limited to E. coli.

\section{AUTHOR CONTRIBUTIONS}

Conceived and designed the experiments: Y-FY and SL. Performed the experiments: SL and QZ. Analyzed the data: SL and ZX. Contributed reagents/materials/analysis tools: SL, QZ, and ZX. Wrote the paper: SL and Y-FY.

\section{ACKNOWLEDGMENTS}

This work was supported by grants from the State Key Development Programs for Basic Research of China (973 Program No. 2015CB554203), Key Research and Development Project of China (No. 2016YFA0500600), the National Natural Science Foundation of China (No. 31270173, No. 31070114, No. 31600033 and No. 81361120383), the Program for Professor of Special Appointment (Eastern Scholar) at Shanghai Institutions of Higher Learning.

\section{SUPPLEMENTARY MATERIAL}

The Supplementary Material for this article can be found online at: http://journal.frontiersin.org/article/10.3389/fmicb. 2017.00699/full\#supplementary-material

Duderstadt, K. E., Chuang, K., and Berger, J. M. (2011). DNA stretching by bacterial initiators promotes replication origin opening. Nature 478, 209-213. doi: $10.1038 /$ nature 10455

Fujimitsu, K., Senriuchi, T., and Katayama, T. (2009). Specific genomic sequences of $E$. coli promote replicational initiation by directly reactivating ADP-DnaA. Genes Dev. 23, 1221-1233. doi: 10.1101/gad.1775809

Grimwade, J. E., Ryan, V. T., and Leonard, A. C. (2000). IHF redistributes bound initiator protein, DnaA, on supercoiled oriC of Escherichia coli. Mol. Microbiol. 35, 835-844. doi: 10.1046/j.1365-2958.2000.01755.x

Hwang, D. S., and Kornberg, A. (1992). Opening of the replication origin of Escherichia coli by DnaA protein with protein HU or IHF. J. Biol. Chem. 267, 23083-23086.

Katayama, T., Ozaki, S., Keyamura, K., and Fujimitsu, K. (2010). Regulation of the replication cycle: conserved and diverse regulatory systems for DnaA and oriC. Nat. Rev. Microbiol. 8, 163-170. doi: 10.1038/nrmicro2314

Kawakami, H., Keyamura, K., and Katayama, T. (2005). Formation of an ATPDnaA-specific initiation complex requires DnaA Arginine 285, a conserved motif in the AAA+ protein family. J. Biol. Chem. 280, 27420-27430. doi: 10.1074/jbc.M502764200

Kornberg, A., and Baker, T. A. (1992). DNA Replication. New York, NY: W. H. Freeman. 
Kuhn, M. L., Zemaitaitis, B., Hu, L. I., Sahu, A., Sorensen, D., Minasov, G., et al. (2014). Structural, kinetic and proteomic characterization of acetyl phosphatedependent bacterial protein acetylation. PLoS ONE 9:e94816. doi: 10.1371/ journal.pone.0094816

Langer, U., Richter, S., Roth, A., Weigel, C., and Messer, W. (1996). A comprehensive set of DnaA-box mutations in the replication origin, oriC, of Escherichia coli. Mol. Microbiol. 21, 301-311. doi: 10.1046/j.1365-2958.1996. 6481362.x

Liang, W., and Deutscher, M. P. (2012). Post-translational modification of RNase R is regulated by stress-dependent reduction in the acetylating enzyme Pka (YfiQ). RNA 18, 37-41. doi: 10.1261/rna.030213.111

Ma, Q., and Wood, T. K. (2011). Protein acetylation in prokaryotes increases stress resistance. Biochem. Biophys. Res. Commun. 410, 846-851. doi: 10.1016/j.bbrc. 2011.06.076

McGarry, K. C., Ryan, V. T., Grimwade, J. E., and Leonard, A. C. (2004). Two discriminatory binding sites in the Escherichia coli replication origin are required for DNA strand opening by initiator DnaA-ATP. Proc. Natl. Acad. Sci. U.S.A. 101, 2811-2816. doi: 10.1073/pnas.0400340101

Messer, W. (2002). The bacterial replication initiator DnaA. DnaA and oriC, the bacterial mode to initiate DNA replication. FEMS Microbiol. Rev. 26, 355-374.

Messer, W., and Weigel, C. (1997). DnaA initiator-also a transcription factor. Mol. Microbiol. 24, 1-6. doi: 10.1046/j.1365-2958.1997.3171678.x

Mizushima, T., Takaki, T., Kubota, T., Tsuchiya, T., Miki, T., Katayama, T., et al. (1998). Site-directed mutational analysis for the ATP binding of DnaA protein. Functions of two conserved amino acids (Lys-178 and Asp-235) located in the ATP-binding domain of DnaA protein in vitro and in vivo. J. Biol. Chem. 273, 20847-20851. doi: 10.1074/jbc.273.33.20847

Mott, M. L., and Berger, J. M. (2007). DNA replication initiation: mechanisms and regulation in bacteria. Nat. Rev. Microbiol. 5, 343-354. doi: 10.1038/ nrmicro1640

Neumann, H., Peak-Chew, S. Y., and Chin, J. W. (2008). Genetically encoding $\mathrm{N}$ (epsilon)-acetyllysine in recombinant proteins. Nat. Chem. Biol. 4, 232-234. doi: $10.1038 /$ nchembio.73

O'Donnell, M. (2006). Replisome architecture and dynamics in Escherichia coli. J. Biol. Chem. 281, 10653-10656. doi: 10.1074/jbc.R500028200

Ozaki, S., Kawakami, H., Nakamura, K., Fujikawa, N., Kagawa, W., Park, S. Y., et al. (2008). A common mechanism for the ATP-DnaA-dependent formation of open complexes at the replication origin. J. Biol. Chem. 283, 8351-8362. doi: 10.1074/jbc.M708684200

Qin, R., Sang, Y., Ren, J., Zhang, Q., Li, S., Cui, Z., et al. (2016). The bacterial two-hybrid system uncovers the involvement of acetylation in regulating of Lrp activity in Salmonella Typhimurium. Front. Microbiol. 7:1864. doi: 10.3389/ fmicb.2016.01864

Ren, J., Sang, Y., Tan, Y., Tao, J., Ni, J., Liu, S., et al. (2016). Acetylation of lysine 201 inhibits the DNA-binding ability of PhoP to regulate Salmonella virulence. PLoS Pathog. 12:e1005458. doi: 10.1371/journal.ppat.1005458

Ren, Q., and Gorovsky, M. A. (2001). Histone H2A.Z acetylation modulates an essential charge patch. Mol. Cell 7, 1329-1335. doi: 10.1016/S1097-2765(01) 00269-6

Rozgaja, T. A., Grimwade, J. E., Iqbal, M., Czerwonka, C., Vora, M., and Leonard, A. C. (2011). Two oppositely oriented arrays of low-affinity recognition sites in oriC guide progressive binding of DnaA during Escherichia coli pre-RC assembly. Mol. Microbiol. 82, 475-488. doi: 10.1111/j.1365-2958.2011.07827.x

Ryan, V. T., Grimwade, J. E., Camara, J. E., Crooke, E., and Leonard, A. C. (2004). Escherichia coli prereplication complex assembly is regulated by dynamic interplay among Fis. IHF and DnaA. Mol. Microbiol. 51, 1347-1359. doi: 10.1046/j.1365-2958.2003.03906.x
Sang, Y., Ren, J., Ni, J., Tao, J., Lu, J., and Yao, Y. F. (2016). Protein acetylation is involved in Salmonella enterica Serovar Typhimurium virulence. J. Infect. Dis. 213, 1836-1845. doi: 10.1093/infdis/jiw028

Schaper, S., and Messer, W. (1995). Interaction of the initiator protein DnaA of Escherichia coli with its DNA target. J. Biol. Chem. 270, 17622-17626. doi: 10.1074/jbc.270.29.17622

Schilling, B., Christensen, D., Davis, R., Sahu, A. K., Hu, L. I., WalkerPeddakotla, A., et al. (2015). Protein acetylation dynamics in response to carbon overflow in Escherichia coli. Mol. Microbiol. 98, 847-863. doi: 10.1111/mmi. 13161

Sekimizu, K., Bramhill, D., and Kornberg, A. (1987). ATP activates dnaA protein in initiating replication of plasmids bearing the origin of the E. coli chromosome. Cell 50, 259-265. doi: 10.1016/0092-8674(87)90221-2

Skarstad, K., and Boye, E. (1994). The initiator protein DnaA: evolution, properties and function. Biochim. Biophys. Acta 1217, 111-130. doi: 10.1016/01674781(94)90025-6

Speck, C., and Messer, W. (2001). Mechanism of origin unwinding: sequential binding of DnaA to double- and single-stranded DNA. EMBO J. 20, 1469-1476. doi: 10.1093/emboj/20.6.1469

Starai, V. J., Celic, I., Cole, R. N., Boeke, J. D., and Escalante-Semerena, J. C. (2002). Sir2-dependent activation of acetyl-CoA synthetase by deacetylation of active lysine. Science 298, 2390-2392. doi: 10.1126/science.1077650

Starai, V. J., and Escalante-Semerena, J. C. (2004). Identification of the protein acetyltransferase (Pat) enzyme that acetylates acetyl-CoA synthetase in Salmonella enterica. J. Mol. Biol. 340, 1005-1012. doi: 10.1016/j.jmb.2004. 05.010

Tu, S., Guo, S. J., Chen, C. S., Liu, C. X., Jiang, H. W., Ge, F., et al. (2015). YcgC represents a new protein deacetylase family in prokaryotes. Elife 4:e05322. doi: 10.7554/eLife.05322

Wagner, G. R., and Payne, R. M. (2011). Mitochondrial acetylation and diseases of aging. J. Aging Res. 2011, 234875. doi: 10.4061/2011/234875

Wang, Q., Zhang, Y., Yang, C., Xiong, H., Lin, Y., Yao, J., et al. (2010). Acetylation of metabolic enzymes coordinates carbon source utilization and metabolic flux. Science 327, 1004-1007. doi: 10.1126/science.1179687

Wang, Y., Cen, X. F., Zhao, G. P., and Wang, J. (2012). Characterization of a new GlnR binding box in the promoter of amtB in Streptomyces coelicolor inferred a PhoP/GlnR competitive binding mechanism for transcriptional regulation of amtB. J. Bacteriol. 194, 5237-5244. doi: 10.1128/JB.00989-12

Weinert, B. T., Iesmantavicius, V., Wagner, S. A., Scholz, C., Gummesson, B., Beli, P., et al. (2013). Acetyl-phosphate is a critical determinant of lysine acetylation in E. coli. Mol. Cell 51, 265-272. doi: 10.1016/j.molcel.2013. 06.003

Zhang, Q., Zhou, A., Li, S., Ni, J., Tao, J., Lu, J., et al. (2016). Reversible lysine acetylation is involved in DNA replication initiation by regulating activities of initiator DnaA in Escherichia coli. Sci. Rep. 6:30837. doi: 10.1038/srep 30837

Conflict of Interest Statement: The authors declare that the research was conducted in the absence of any commercial or financial relationships that could be construed as a potential conflict of interest.

Copyright (c) 2017 Li, Zhang, Xu and Yao. This is an open-access article distributed under the terms of the Creative Commons Attribution License (CC BY). The use, distribution or reproduction in other forums is permitted, provided the original author(s) or licensor are credited and that the original publication in this journal is cited, in accordance with accepted academic practice. No use, distribution or reproduction is permitted which does not comply with these terms. 